\title{
An analytic hierarchy process approach for the security assessment of high speed railway construction sites
}

Conference Paper · June 2017

DOI: $10.1201 / 9781315210469-189$

\section{CITATIONS}

0

5 authors, including:

\section{Roberto Setola}

Università Campus Bio-Medico di Roma 194 PUBLICATIONS 1,454 CITATIONS

SEE PROFILE
READS

38

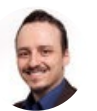

Marco Tesei

Università Campus Bio-Medico di Roma 10 PUBLICATIONS 9 CITATIONS

SEE PROFILE

Some of the authors of this publication are also working on these related projects:

Project

REF - Requirements engineering framework View project 


\title{
An Analytic Hierarchy Process Approach for the Security Assessment of High Speed Railway Construction Sites
}

\author{
R. Setola, M. De Maggio, G. Natale, M. Tesei \\ Università Campus Bio-Medico di Roma, Italy \\ Consorzio NITEL, Italy
}

E. Zio

Chair System Science and Energy Challenge, Fondation Electricité de France (EDF), CentraleSupélec, Université Paris-Saclay, Chatenay-Malabry, France

Politecnico di Milano, Italy

ABSTRACT: The construction of High Speed Railways often faces an increasing number of obstacles because of the opposition of the local population and the environmental activist movements, or similar ideological groups. Therefore, the need of identifying the optimal location for the construction sites to minimize both the overall costs of the project and the risk of negative consequences is a key point, since security in construction sites has become a critical issue.

In this paper, we illustrate a methodology specifically developed to support the analysis for the optimal location of the construction sites for the $57 \mathrm{~km}$ tunnel of the high speed railway Lyon-Turin. To perform the security assessment, we adopted the Analytic Hierarchy Process (AHP), properly tailored to manage the peculiarities of this specific security problem. The proposed framework has been used to perform an iterative analysis aimed at identifying the most suitable construction site locations, under given objectives and constraints.

\section{INTRODUCTION}

\subsection{Aim and Context}

The construction of High Speed Railways often faces an increasing number of difficulties because of the opposition of the local population, the environmental activists and similar ideological groups (Setola, 2015). Then, the aim of a security assessment must be to identify the optimal location for the construction sites, to minimize the potential negative consequences of protests on the population, the workers, the businesses and the costs of the construction project. The topic was addressed only recently in the literature. In (Sadi and El-Rayes, 2010) it is analyzed how to include the security issue in the planning of construction site layouts. The problem is treated in (Li et al. 2015) as a bilevel and multiobject optimization problem. An overview about construction site security is reported in (Arata, 2006) while (Boba and Santos, 2008) analyses the phenomenon of thefts inside construction sites.

In this paper, we illustrate a methodology developed for the optimization of the location of the construction sites for the $57 \mathrm{~km}$ tunnel of the high speed railway Lyon-Turin. This $€ 8$ billion mining engineering project has been suffering a strong opposition, especially on the Italian side, which has caused more than 1,000 injured in the last 4 years, a considerable increment in the costs and a large delay on the time scheduling of the project.
During the investigation phase for the approval of the final project, the Italian Government has requested a detailed risk assessment of the 10 planned construction sites, regarding to safety and security. It also asked to consider alternative locations to improve the safety of workers and population.

To perform such assessment, we adopted the Analytic Hierarchy Process (AHP) (Saaty, 1990, Zio, 1996), properly tailored to manage the specific security issues. To identify the most secure solution, we compared different construction site configurations, that have been evaluated with regard to how much the site is considered a valuable target by the opponents and to what the potential consequences of an attack might be.

The methodology is used to perform a comparative analysis of the different configurations, with the aim to identify which are the most suitable ones. The evaluation is made quite hard by the presence of several feasibility constraints in the possible alternative construction sites. Moreover, the establishment of a new site would impact the other existing ones, in terms of work allocation, organization and timescheduling.

To systematically manage the evaluation process, a risk analysis framework has been adopted to exclude inadequate solutions at the early stages and to focus the design process on those solutions considered the most suitable. Obviously, at the early stages only qualitative information is available, whereas in the latter stages more detailed information and quan- 
titative data are used. Thus, the security assessment framework must be able to manage different levels of granularity and details.

For this reason, the proposed framework starts modelling the different elements involved in the assessment using qualitative information (soft data), which are subsequently decomposed in tangible quantities (hard data), following an approach developed for software engineering requirements (Donzelli and Setola, 2007).

In this paper, we illustrate the methodological framework adopted for the security assessment of the sites. For obvious reasons, no details are provided about the sites and all data have been conveniently camouflaged.

\subsection{Paper Organization}

The paper is arranged as follows: in Section 2, the Lyon-Turin high speed railway project is briefly described, together with the related security problems occurred in the last few years. The methodology adopted for the security assessment is described in Section 3, while Section 4 shows some results, and some closing comments are collected in Section 5.

\section{LYON-TURIN HIGH SPEED RAILWAY PROJECT}

\subsection{The project}

The construction of the high speed railway LyonTurin is part of the Mediterranean Corridor, one of the nine Trans-European transport Networks (TENT) lunched by the European Commission with the aim to improve European mobility (see Figure 1 and https://ec.europa.eu/transport/themes/infrastructure en for more details). Specifically, the Mediterranean Corridor is going to be about $3,000 \mathrm{~km}$ long, crossing the Southern Europe from West (Spain) to East (Russia) through France, Italy, Slovenia, Croatia and Hungary.

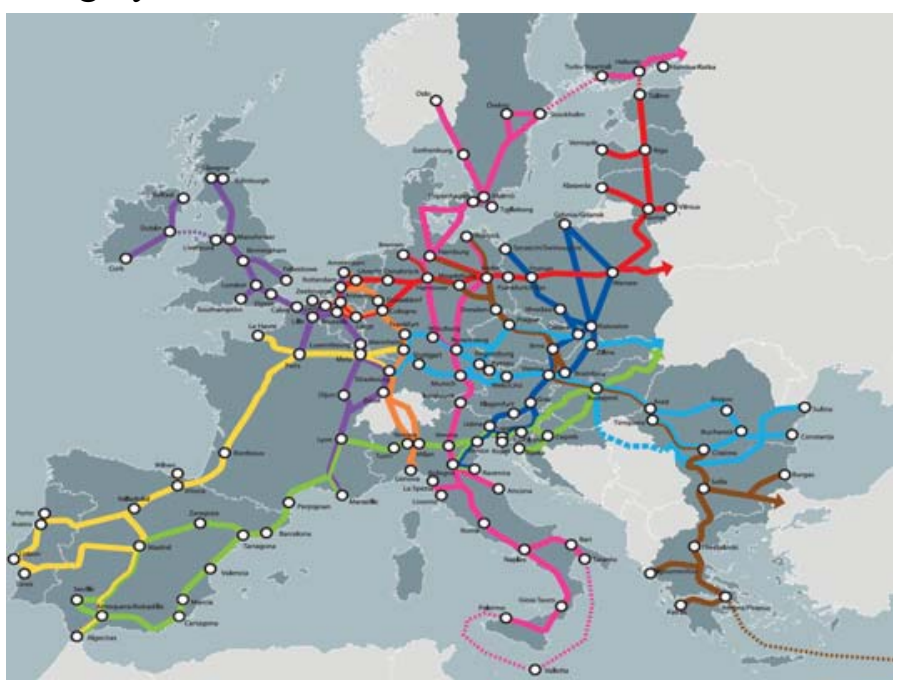

Figure 1: TEN-T Corridors.
The Mediterranean Corridor will cross the Alps from Lyon to Turin. As the old $6 \mathrm{~km}$ Frejus tunnel lies $1,000 \mathrm{~m}$ above sea level with a grade profile up to $33 \%$, there is the need to replace it with a new one. As showed in Figure 2, the new tunnel is going to be $57 \mathrm{~km}$ long and it will connect Susa (Italy) to St Jean-de-Maurienne (France) at an altitude of about $500 \mathrm{~m}$ and with a maximum grade profile of $12,5 \%$. Trains will be able to operate at speeds up to 220 $\mathrm{km} / \mathrm{h}$. The tunnel has been estimated to cost $€ 8$ billion and it is expected to be fully operational by 2030. Once finished, it will be the longest railway tunnel in the world.

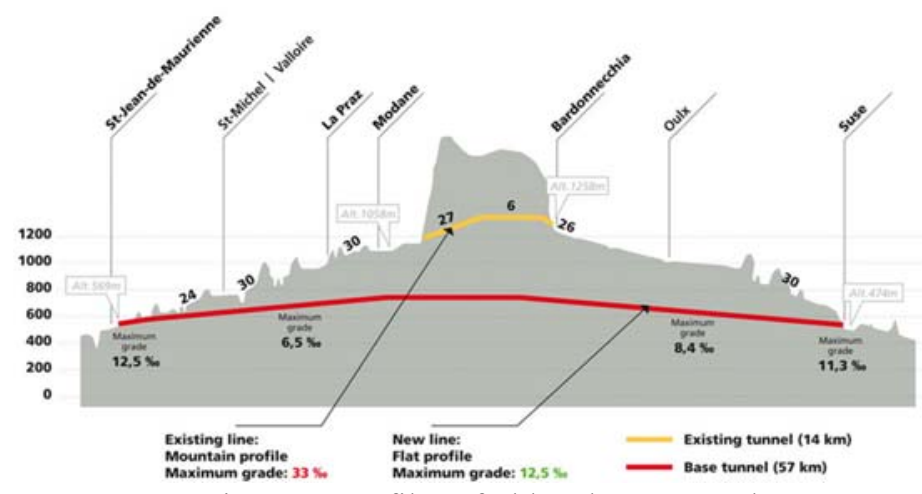

Figure 2. Profiles of old and new tunnels

The tunnel construction will be carried out by TELT SAS, a company specifically established by the French and the Italian governments for the construction and the management of the tunnel. All the preliminarily geognostic tests have been performed and at the end of 2016 the French and Italian parliaments approved the agreement to start constructing.

\subsection{Opposition to the project}

The project has suffered a strong opposition from the local population, especially on the Italian side, because it is considered unnecessary, useless, too expensive and dangerous for the environment. This opposition has been partially led by extremist groups, which tried to obtain large visibility and authority under the "NoTav" flag. These groups promoted a violent opposition against the tunnel, arranged many sabotages and attacks against the construction sites and the companies involved in the construction activities. In the period from 2012 to 2016, about 200 violent attacks against the site La Maddalena (the Italian site of the geognostic test tunnel) have occurred. These attacks have caused more than 1,000 injuries in law enforcement officers, a large delay in the construction activities, a strong concern among the population (the tourism in the area dropped) and they have forced the adoption of an increased number of security measures for the construction site (with a significant cost increase). 


\subsection{The aim of the security assessment}

During the process for the final approval of the tunnel, because of such violent opposition, the Italian Government has asked for a detailed analysis of the security risks for all the planned construction sites, and to consider alternative locations for those sites to reduce security risks. This request was quite relevant because originally security issues were not taken into account in choosing the construction site locations; it was only considered the maximum efficiency in the operation of the construction activities.

The goal of the security assessment has been to compare the risks of the already planned sites with those of the alternative locations, with the aim of identifying the best configurations, i.e. those that minimize the security risks both for population, workers and business (operation) continuity.

The assessment and comparison tasks have been made difficult by the several constraints to be considered to identify alternative locations for the construction sites. Moreover, introducing a new site impacts the other existing ones, with respect to work activities, allocation, organization and timescheduling. This implies the re-design of the layout of all sites, with consequent changes in the whole security assessment. This entails a global consideration of the overall "construction sites configuration", i.e. considering globally the security issues of all the involved construction sites (and the logistic aspects related to their operations).

To this end, as illustrated more in detail in the next section, the security risk of each construction site is modelled in terms of its "sensibility" and the potential "consequences" of a sabotage/attack. These features are iteratively decomposed, on the base of the available information, into elementary and measurable quantities parameters. The different quantities are aggregated using the Analytic Hierarchy Process (AHP) methodology to estimate the level of security risk of each site. Moreover, to evaluate the security risk of each construction site configuration the values of the different sites in the configuration are aggregated considering the time duration of their operations too.

To focus the analysis effort only on the most suitable configuration solutions, an iterative process has been adopted to discard the unfeasible configurations at the early stages.

\section{THE METHODOLOGY}

\subsection{The proposed approach}

The analysis of the factors involved in the security risks to which a construction site is exposed is struc- tured by the AHP in a top-down formulation. In the first stage, the analysis is performed using abstract and qualitative properties of the construction sites, which are decomposed at a later stage into their measurable constituents. Indeed, at the early stage of the analysis of an alternative construction site the information and data available are very limited, i.e. information and data on the geographic location of the area, with little details about perimeters, layouts, working and operation tasks, etc. At the same time, as mentioned above, introducing such a new site in the overall configuration modifies the organization of some other sites in the configuration and produces a limited availability of preliminary information (e.g. "the presence of this new site will reduce a little the activities in the other sites", etc.).

Since it is impossible to design or re-design in detail the layout of all the potential sites in all the possible configurations, the security assessment is performed iteratively to identify and discard the infeasible solutions.

The level of security risk associated to any site is modelled in terms of (see Figure 4):

- Sensibility. which provides a measure of the belief that someone may perform a sabotage/attack against the site.

- Impact: which provides an estimation of the possible consequences of a sabotage/attack.

As mentioned in the introduction of this paper we illustrate the approach used to estimate the sensibility of each site. This quantity is decomposed into:

- Attractiveness. How much the site is considered valuable as a possible target from the opponents. In other words, the attractiveness quantifies the benefits that the enemy can achieve by finalizing the action against the site.

- Vulnerability. How much easy (or hard) is to accomplish a sabotage/attack against the site. It depends on the intrinsic characteristics of the site and on the attacker's technical capabilities.

These parameters depend both on macro-scale data, i.e. on characteristic of the area where the site is located, and on the peculiarities of the site, i.e. extension, morphology, activities, etc. Notice that for the problem at hand the experts consider the vulnerability more relevant than the attractiveness $(>)$.

We asked to the experts to provide a grade for each parameter for all the construction sites using a five-level scale (LOW=1, MEDIUM-LOW, MEDIUM, MEDIUM-HIGH and HIGH=5).

Obviously, at this level of abstraction a rough estimation is given. To improve the accuracy these quantities have been decomposed into their macroparameters. More specifically, the attractiveness has been split up into the following seven parameters:

- Symbolic value of the site. The sites directly related to the base tunnel have a greater symbolic value than the deposit sites. In the same way, the 
sites located in a particular area might be perceived as more valuable than others.

- Visibility. Some sites are highly visible both from population and media point of view; the visibility has a strong impact on the attractiveness of a site because the effect of an action performed against a visible site will be emphasized by the media.

- Expected damage. The larger is the foreseen level of impact of a sabotage/attack the more a site is attractive. The level of impact taken into account in this case is the level assumed by the opponent point of view (which is usually different from the effective level of damage).

- Absence of collateral damage. Since opponents wish to have the support of the population they are very careful not to damage the population itself - and in some cases workers too.

- Accessibility. The more a site is easy to reach (and to run away from) the more it is attractive.

- Context. Some areas of the valley are historically characterized by a strong feeling against the tunnel construction and this feeling resulted in violent movements. In these areas opponents find a broad ideological and logistic support.

- Isolation. If a site is located in a remote area, any illegal action is easy to perform because of the lack of control.

These parameters largely depend on macro-scale data so they can be easily estimated at very early design stage too, with the help of experts using the mentioned five-level scale.

Notice that since the goal of the procedure is not to provide an absolute value of the risk associated to each site but to perform a comparative analysis of the different solutions, the experts can easily provide a good estimation of the different parameters using the site La Maddalena as reference.

\begin{tabular}{|c|c|c|c|c|c|c|c|}
\hline ATTRACTIVENESS & 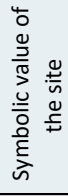 & 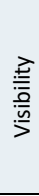 & 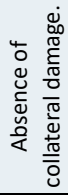 & 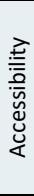 & 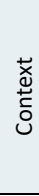 & $\begin{array}{l}\text { 든 } \\
\stackrel{0}{0} \\
\text { 응 } \\
\underline{n}\end{array}$ & 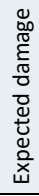 \\
\hline Symbolic value of the site & $\mathrm{x}$ & $\gg$ & $=$ & $>$ & $\gg$ & $\ll$ & $>$ \\
\hline Visibility & $\mathrm{x}$ & $\mathrm{x}$ & $=$ & $=$ & $>$ & $>$ & $>$ \\
\hline $\begin{array}{c}\text { Absence of collateral } \\
\text { damage }\end{array}$ & $x$ & $x$ & $\mathrm{x}$ & $=$ & $=$ & $=$ & $<$ \\
\hline Accessibility & $\mathrm{x}$ & $\mathrm{x}$ & $\mathrm{x}$ & $\mathrm{x}$ & $>$ & $\gg$ & $<$ \\
\hline Context & $x$ & $\mathrm{x}$ & $\mathrm{x}$ & $x$ & $\mathrm{x}$ & $\gg$ & $>$ \\
\hline Isolation & $x$ & $\mathrm{x}$ & $\mathrm{x}$ & $x$ & $x$ & $\mathrm{x}$ & $<<$ \\
\hline Expected damage & $x$ & $x$ & $x$ & $x$ & $x$ & $x$ & $x$ \\
\hline
\end{tabular}

Figure 3. Relative relevance of attractiveness parameters.

To compare the relevance of the different parameters using the AHP approach we asked to the experts to express the relative importance of any cou- ple of parameters using a five-level scale: not relevant at all $(<<$, i.e. $1 / 5)$, slightly relevant $(<$, i.e. $1 / 3)$, equally relevant $(=$, i.e. 1$)$, very relevant $(>$, i.e. 3$)$, extremely relevant $(>>$, i.e. 5). The results are collected in Figure 3.

We are able to better define the estimation of the site attractiveness combining the value of the parameters resulted by applying the AHP method. This new estimation overwrites the previous rough one. The parameters that characterize the vulnerability of a site are more related to the characteristics of the construction site, so we need to consider an iterative process. Hence in the first stages they are evaluated on the base of experts' opinions and then progressively replaced with numerical values calculated on the basis of design data and other information. After that the values of the different quantitative factors are elaborated following the AHP methodology.

Specifically, to estimate the vulnerability we consider that vulnerability of a site depends on its:

- Attackability. How easily the site can be attacked by the opponents.

- Defensibility. How much the site configuration facilitates the protection.

This latter is considered more relevant $(>)$ than attackability and both the parameters are assessed by the experts for each site. To have better defined estimations both parameters are decomposed as follows.

The attackability is split into:

- Accessibility. The easier a site is to reach or to run away from (especially by vehicles) the more attractive it will be for sabotages/attacks.

- Complexity of the construction activities within

the site. The more construction activities are complex, the easier is for the opponents to exploit the site's vulnerabilities.

These parameters, when the relative information is available, are further decomposed as follows:

Accessibility

o Number of roads accessible to vehicles

o Number of pedestrian paths

o Number of accesses to the site

o Morphology of the surrounding (e.g. the construction site is on the top of a hill or at the bottom of a valley)

o Characteristics of the natural surrounding (i.e. dense vegetation instead of grassland)

o Presence of natural barriers (e.g. rivers, cliff walls, etc.)

Complexity of the construction activities within the site.

o Site Logistics: the presence of several operating employees and vehicles in case of complex activities may facilitate the opponents in the making of sabotages/attacks. 
o Number of employees that operate in the site.

o Number of vehicles that operate in the site.

o Number of accesses per day: in case of a high number of accesses both from employees and vehicles, a fast access control is needed. in:

On the other side the Defensibility is decomposed

o Perimeter length: a long perimeter is more complex and expensive to protect than a short one.

o Perimeter morphology: an irregular perimeter is hard to control. This can be further decomposed into:

o Site extension: the larger a site is, the harder its control is.

o Site morphology: irregular altimetry, inside and outside the site, reduces the visibility, facilitating the opponents.

o Presence, of dominant positions that can be used by the opponents as a vantage point to monitor the activities, through objects against the site or to perform other kinds of sabotages/attacks.

Some of the above-mentioned parameters are already measurable quantities, so they are properly converted in a $0-5$ scale (in which 5 is associated with the maximum value with respect to all the sites in all the configuration). The others are further refined in order to decompose them into measurable quantities (we neglect these details for security reasons).

The whole process is illustrated in Figure 4. The high level of hierarchy in the decomposition of the vulnerability is due to the difficulties of the experts to compare heterogeneous quantities, Indeed, we need to aggregate the different elements into more abstract quantities, which become easier to be compared (a different solution to overcome this problem is to consider AHP with incomplete information as proposed in (Oliva et al, 2016).

\section{RESULTS}

The proposed procedure has been used to assess the ten construction sites foreseen in the nominal project. The results are reported in the Figure 5.

The analysis emphasizes that there are two sites with high levels of sensibility and impact (red zone) and other two sites close to the border between the yellow and the red zones. Therefore, these four sites are very critical from the security point of view.

Several alternative solutions have been analyzed for reducing the security risk. In Figure 6, we report the best solution, that contains eleven sites (obtained introducing two new sites and removing one of the planned sites $\left.{ }^{1}\right)$. Specifically, thanks to a complete re-arrangement of the construction activities in all the sites, there is a considerable reduction of the security risk level of each site. By now, only one site is in the red zone of the graph (with a significant reduction of its sensibility and impact with respect to previous configurations) and no one of the sites close to the border between the red and the yellow zones.

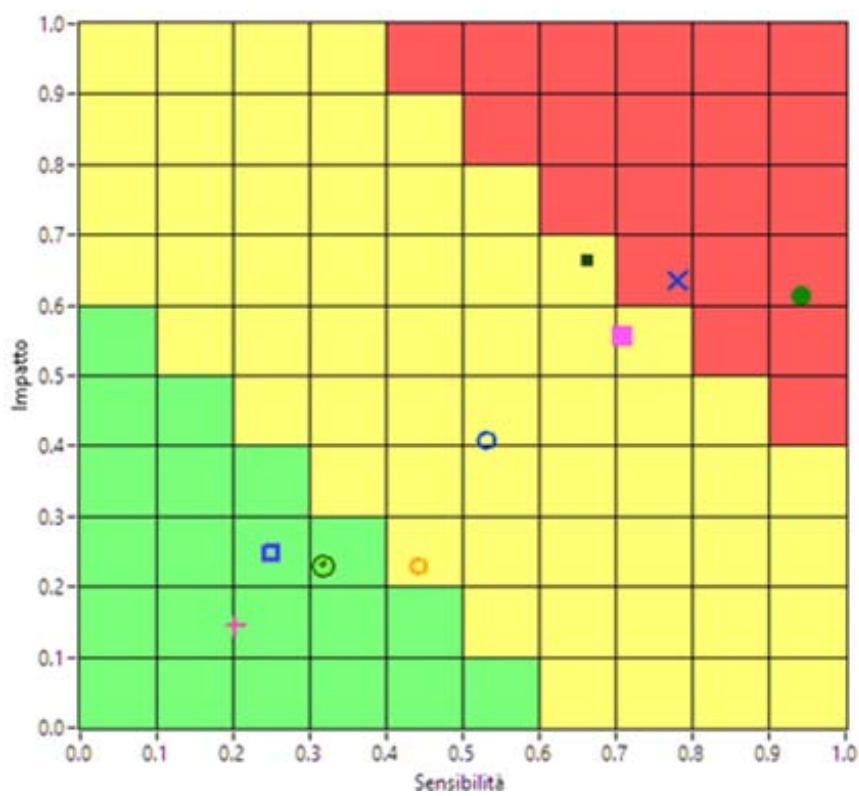

Figure 5. Results of the assessment for the ten sites that constitute the nominal configuration.

\section{CONCLUSIONS}

This paper describes the approach developed by the authors to identify the best location for major works construction sites considering the security issue. This approach is based on an iterative process; for each site the security-relevant parameters are progressively decomposed from abstract and qualitative data into measurable ones.

The analysis of the factors involved in the security risks to which a construction site is exposed is structured by the AHP in a top-down formulation. In the first stage, the analysis is performed using abstract and qualitative properties of the construction sites, which are decomposed at a later stage into their measurable constituents.

The level of security risk associated to any site is modelled in terms of Sensibility and Impact, decomposed in several further indicators evaluated in the following phases of the mentioned iterative process, as described in Section 3.

\footnotetext{
${ }^{1}$ Notice that each symbol is related to a peculiar site.
} 


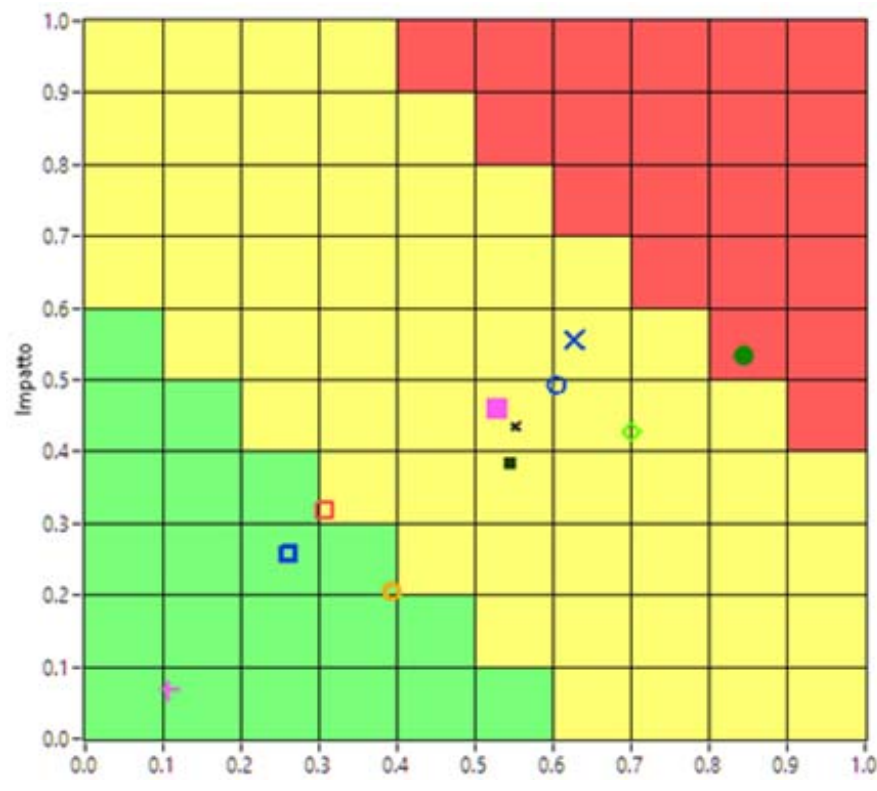

Figure 6. Results of the risk assessment for the optimal configuration.

The procedure has been used to identify the best locations for the construction sites of the new LyonTurin high speed railway tunnel.

\section{REFERENCES}

Donzelli, P., \& Setola, R. (2007). "Identifying and evaluating risks related to enterprise dependen- cies: a practical goal-driven risk analysis framework". International Journal of Risk Assessment and Management, 7(8), 1120-1137.

Oliva, G, Panzieri S., and Setola R. "Fuzzy dynamic input-output inoperability model". International Journal of Critical Infrastructure Protection 4.3 (2011): 165-175

Saaty, T. L. (1990). "How to make a decision: the analytic hierarchy process". European journal of operational research, 48(1), 9-26.

Setola, R., Sforza, A., Vittorini, V., \& Pragliola, C. (Eds.). (2015). Railway Infrastructure Security (Vol. 27). Springer. Zio, E. "On the use of the analytic hierarchy process in the aggregation of expert judgments." Reliability Engineering \& System Safety 53.2 (1996): 127-138. Said, H, and K. El-Rayes. "Optimizing the planning of construction site security for critical infrastructure projects." Automation in Construction 19.2 (2010): 221-234.

Li, Z., Shen, W., Xu, J., \& Lev, B. (2015). "Bilevel and multi-objective dynamic construction site layout and security planning". Automation in Construction, 57, 1-16.

Arata, M. J. (2006). "Construction site security". McGraw-Hill.

Boba, R., \& Santos, R. (2008). "A review of the research, practice, and evaluation of construction site theft occurrence and prevention: directions for future research". Security Journal, 21(4), 246263.

G. Oliva, R. Setola and A. Scala, "Sparse and Distributed Analytic Hierarchy Process", Automatica (submitted in October 2016).

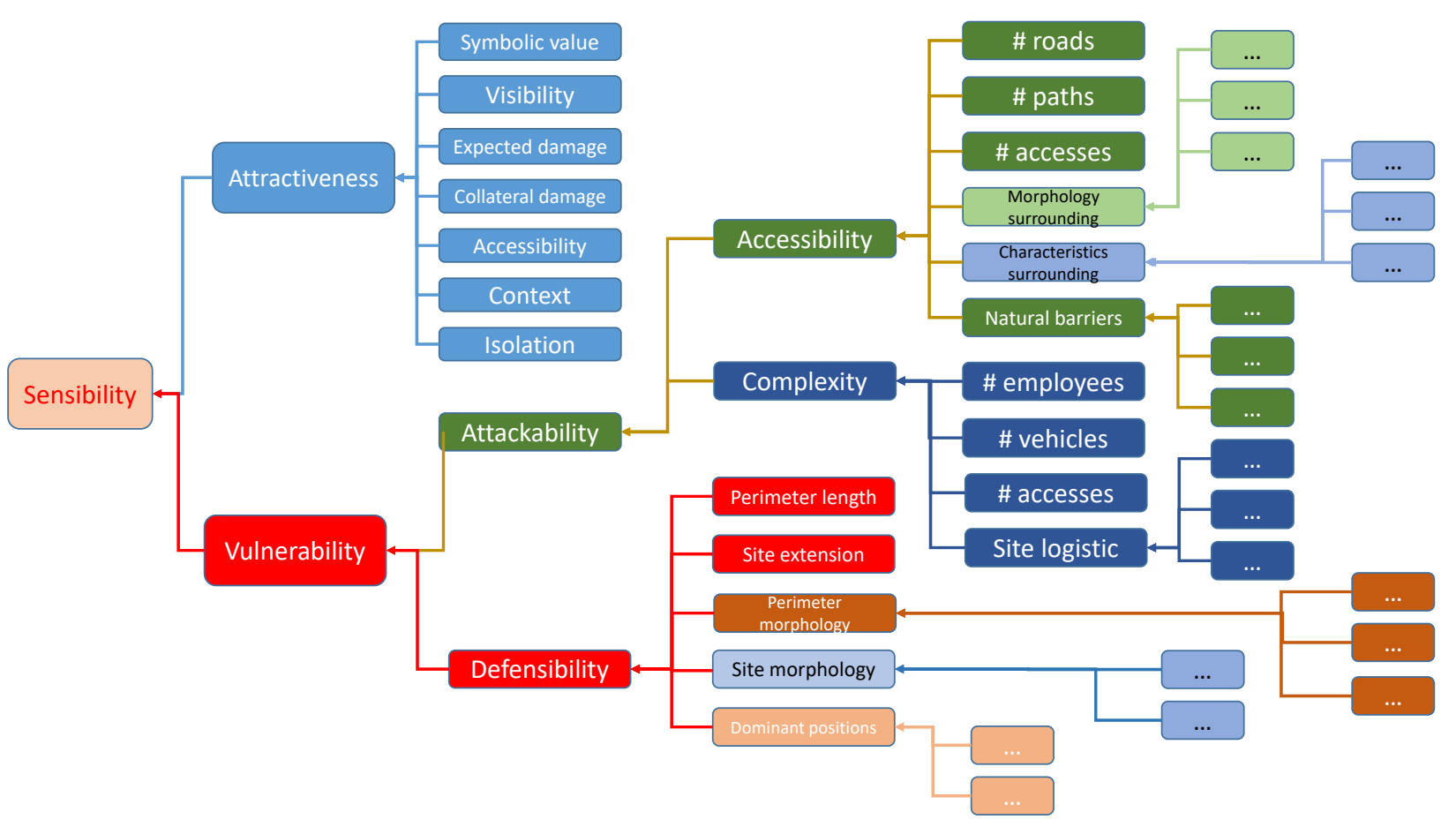

Figure 4. Decomposition of the sensibility parameter. 